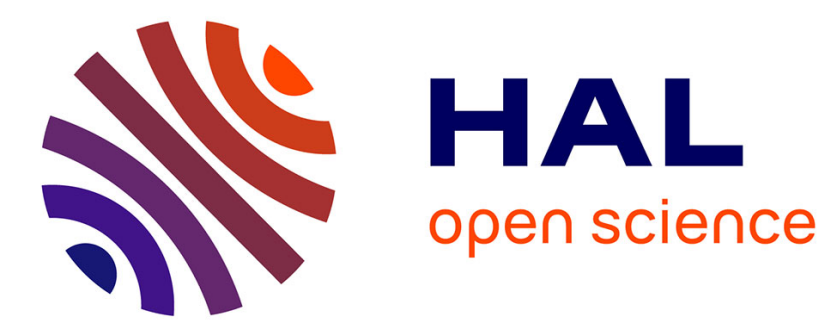

\title{
Numerical simulation of two-phase inertial flow in heterogeneous porous media
}

Azita Ahmadi-Senichault, Ali Akbar Abbasian Arani, Didier Lasseux

\section{To cite this version:}

Azita Ahmadi-Senichault, Ali Akbar Abbasian Arani, Didier Lasseux. Numerical simulation of twophase inertial flow in heterogeneous porous media. Transport in Porous Media, 2010, 84 (1), pp.177200. 10.1007/s11242-009-9491-1 . hal-01174073

\section{HAL Id: hal-01174073 \\ https://hal.science/hal-01174073}

Submitted on 8 Jul 2015

HAL is a multi-disciplinary open access archive for the deposit and dissemination of scientific research documents, whether they are published or not. The documents may come from teaching and research institutions in France or abroad, or from public or private research centers.
L'archive ouverte pluridisciplinaire HAL, est destinée au dépôt et à la diffusion de documents scientifiques de niveau recherche, publiés ou non, émanant des établissements d'enseignement et de recherche français ou étrangers, des laboratoires publics ou privés. 


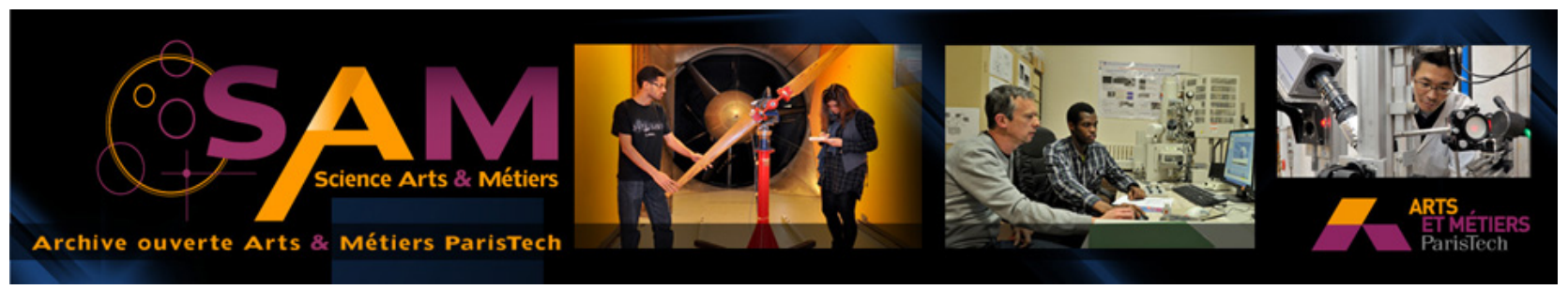

Science Arts \& Métiers (SAM)

is an open access repository that collects the work of Arts et Métiers ParisTech researchers and makes it freely available over the web where possible.

This is an author-deposited version published in: http://sam.ensam.eu

Handle ID: .http://hdl.handle.net/10985/9727

\section{To cite this version :}

Azita AHMADI, Ali Akbar ABBASIAN ARANI, Didier LASSEUX - Numerical simulation of twophase inertial flow in heterogeneous porous media - Transport in Porous Media - Vol. 84, $\mathrm{n}^{\circ} 1$, p. $177-200-2010$ 


\title{
Numerical Simulation of Two-Phase Inertial Flow in Heterogeneous Porous Media
}

\author{
Azita Ahmadi • Ali Akbar Abbasian Arani • \\ Didier Lasseux
}

\begin{abstract}
In this study, non-Darcy inertial two-phase incompressible and non-stationary flow in heterogeneous porous media is analyzed using numerical simulations. For the purpose, a 3D numerical tool was fully developed using a finite volume formulation, although for clarity, results are presented in 1D and 2D configurations only. Since a formalized theoretical model confirmed by experimental data is still lacking, our study is based on the widely used generalized Darcy-Forchheimer model. First, a validation is performed by comparing numerical results of the saturation front kinetics with a semi-analytical solution inspired from the Buckley-Leverett model extended to take into account inertia. Second, we highlight the importance of inertial terms on the evolution of saturation fronts as a function of a suitable Reynolds number. Saturation fields are shown to have a structure markedly different from the classical case without inertia, especially for heterogeneous media, thereby, emphasizing the necessity of a more complete model than the classical generalized Darcy's one when inertial effects are not negligible.
\end{abstract}

Keywords Inertial two-phase flow $\cdot$ Heterogeneous porous media $\cdot$ Numerical simulations . Generalized Darcy-Forchheimer model

\section{List of Symbols}

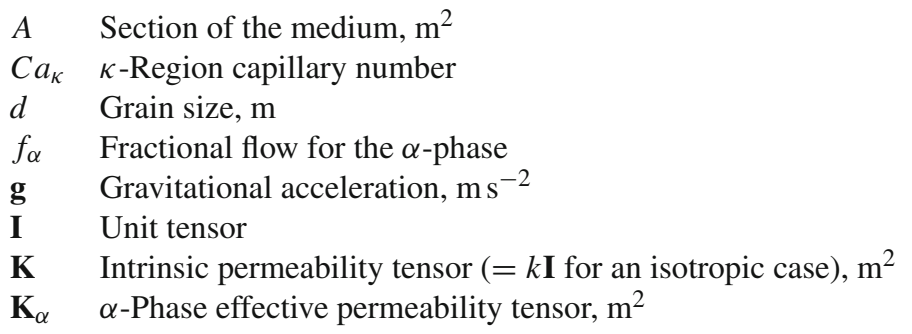

\footnotetext{
A. Ahmadi · A. A. Abbasian Arani · D. Lasseux (凶)

TREFLE, UMR CNRS 8508, Arts et Métiers ParisTech, University Bordeaux 1, 33405 Talence Cedex, France

e-mail: didier.lasseux@ensam.eu
} 


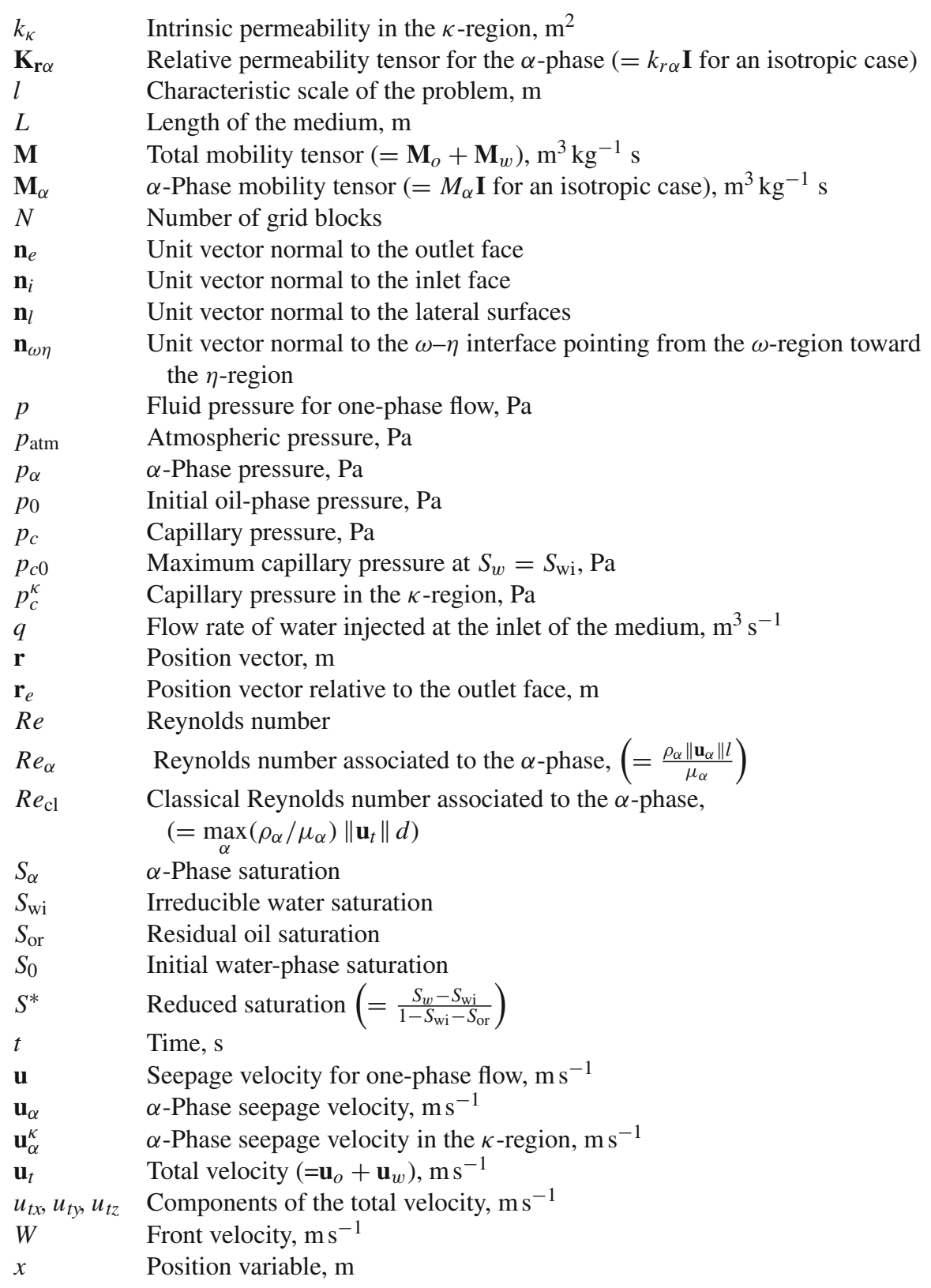

\section{Greek Letters}

$\beta_{\alpha} \quad \alpha$-Phase effective inertial resistance tensor $\left(=\beta_{\alpha} \mathbf{I}\right.$ for the isotropic case $), \mathrm{m}^{-1}$

$\beta \quad$ Intrinsic inertial resistance factor, $\mathrm{m}^{-1}$

$\beta_{r \alpha} \quad \alpha$-Phase relative inertial resistance tensor $\left(=\beta_{r \alpha} \mathbf{I}\right.$ for the isotropic case $)$

$\beta_{\kappa} \quad$ Intrinsic inertial resistance factor for the $\kappa$-region, $\mathrm{m}^{-1}$

$\Gamma_{\omega \eta} \quad$ Interface between the $\omega$-region and the $\eta$-region, $\mathrm{m}^{2}$ 


$\begin{array}{ll}\Delta t & \text { Time step, } \mathrm{s} \\ \Delta x, \Delta y, \Delta z & \text { Grid sizes in the } x, y \text { and } \mathrm{z} \text { directions, } \mathrm{m} \\ \varepsilon & \text { Porosity } \\ \mu_{\alpha} & \alpha \text {-Phase dynamic viscosity, Pas } \\ \rho_{\alpha} & \alpha \text {-Phase density, } \mathrm{kg} / \mathrm{m}^{3} \\ \sigma & \text { Interfacial tension, } \mathrm{N} \mathrm{m}^{-1} \\ \xi, \gamma, \theta & \text { Constant exponents } \\ \tau & \text { Tortuosity }\end{array}$

\section{Introduction}

Under certain conditions such as flow in the near-wellbore region and/or in very permeable reservoirs, hydrocarbon recovery by water flooding or gas injection can lead to high flow rates involving significant inertial effects-but not turbulence-for which the classical description of two-phase flow in porous media by the generalized Darcy's law is no longer valid (Tek et al. 1962; Scheidegger 1972; Katz and Lee 1990; Kalaydjian et al. 1996). This type of flow is also under concern in chemical engineering applications during two-phase flow in packedbed reactors, for instance. As for one-phase flow, inertia is expected to cause extra resistance to flow in each phase leading to an erroneous pressure gradient to velocity relationship while using generalized Darcy's law. Consequently, this extra friction may cause significant modifications of saturation fields and, as shown later, of the breakthrough time. It is, therefore, necessary to call upon a more complete flow model in which inertial effects are taken into account by introducing an additional term in the momentum balance equations classically used for creeping flow. In order to analyze the impact of inertia on macroscopic observable quantities such as saturation, flow rate, and pressure drop, numerical simulations of unsteady two-phase inertial flow are required. So far, very few studies addressing this issue have been reported in contrast to inertial one-phase flow that has concentrated more significant efforts from both theoretical and numerical points of view (Mei and Auriault 1991; Whitaker 1996; Kim and Park 1999; Fourar et al. 2005; Mazaheri et al. 2005; Khashan et al. 2006). Moreover, in most existing numerical investigations, special cases such as steady-state flow without capillary pressure in a cylindrical configuration (Jamiolahmady et al. 2006) or 1D frontal displacement based on Muskat's model assuming piston-like displacement coupled with heat transfer (Sanchez et al. 2005) are considered.

In this study, two-phase, incompressible, non-stationary flow in a non-deformable heterogeneous porous media is considered with Reynolds numbers high enough to justify the use of a model including inertial terms. The physical model used here is the generalized DarcyForchheimer model (Schulenberg and Muller 1987; Wang et al. 1999). Heterogeneous media composed of several homogeneous regions each having isotropic transport properties at the Darcy scale are studied. Without restricting the generality of the development, the wetting and non-wetting phases are distinguished by the indices "w" and "o" referring to water and oil, respectively, and focus is laid upon imbibition only.

\section{Governing Equations}

For sufficiently high flow rates, Darcy's law does not describe correctly the momentum conservation, due to the existence of significant inertial effects. These effects can be taken 
into account by adding a corrective term to Darcy's equation (see, for instance, Ergun 1952 or Hubbert 1956). The problem arising from the presence of significant inertial effects is complex from the point of view of scaling. Some attempts on the formalization of macroscopic models for single-phase flow were undertaken (Mei and Auriault 1991; Whitaker 1996; Skjetne and Auriault 1999). For the two-phase flow case, a theoretical formalization of a model at the Darcy scale has recently been proposed (Lasseux et al. 2008). In the absence of further numerical analysis and confrontation with experimental data, the generalized DarcyForchheimer equation that is classically used in the literature is retained in this study. It is based on the empirical Darcy-Forchheimer model for single-phase flow (Forchheimer 1901) on the one hand and on a generalization of this model to the two-phase flow case in a similar way to that originally followed for flow in the Darcy regime on the other hand. The resulting momentum balance equation involves a quadratic correction of the velocity and is written as $(\alpha=o, w)$

$$
-\left(\nabla p_{\alpha}-\rho_{\alpha} \mathbf{g}\right)=\mu_{\alpha} \mathbf{K}_{\alpha}^{-1} \cdot \mathbf{u}_{\alpha}+\rho_{\alpha}\left\|\mathbf{u}_{\alpha}\right\| \beta_{\alpha} \cdot \mathbf{u}_{\alpha}
$$

in which $\mathbf{u}_{\alpha}, p_{\alpha}, \mu_{\alpha}$, and $\rho_{\alpha}$ are, respectively, the Darcy or seepage velocity, the pressure, the dynamic viscosity, and the density of the $\alpha$-phase; $\mathbf{g}$ is the gravity acceleration. In Eq. 1 , $\mathbf{K}_{\alpha}$ is the $\alpha$-phase effective permeability. It is related to the intrinsic permeability $\mathbf{K}$ by the relation $\mathbf{K}_{\alpha}=\mathbf{K} \cdot \mathbf{K}_{r \alpha}$, where $\mathbf{K}_{r \alpha}$ is the $\alpha$-phase relative permeability tensor that can be explicitly determined as shown in Lasseux et al. (1996). In this study, in addition to scalar intrinsic permeabilities due to isotropy $(\mathbf{K}=k \mathbf{I})$, scalar relative permeabilities $\left(\mathbf{k}_{r \alpha}=k_{r \alpha} \mathbf{I}\right)$ are also considered although this might not be adequate in the general case of anisotropic porous media as reported by Bear et al. (1987) and Ahmadi and Quintard (1995) in which the use of tensorial relative permeabilities was highlighted.

The additional pressure drop resulting from non-Darcy effects due to high flow rate is described by the second term $\left(\rho_{\alpha}\left\|\mathbf{u}_{\alpha}\right\| \beta_{\alpha} \cdot \mathbf{u}_{\alpha}\right)$ on the right-hand side of Eq. 1 . In this term, $\beta_{\alpha}$ is a coefficient (having a priori a tensorial form) generally called as the effective inertial resistance factor or high-velocity flow coefficient or non-Darcy flow coefficient (Geertsma 1974; Liu et al. 1995).

Taking into account isotropy of the medium, one can write $\beta_{\alpha}=\beta_{\alpha} \mathbf{I}=\beta \beta_{r \alpha} \mathbf{I}$, in which $\beta$ is the intrinsic inertial resistance factor of the medium and $\beta_{r \alpha}$ is the $\alpha$-phase relative inertial resistance factor, a nonlinear function of the saturation. Empirical relationships correlating $\beta_{\alpha}$ with porosity, effective permeability, and saturation were proposed in the literature (Geertsma 1974; Evans et al. 1987; Evans and Evans 1988; Liu et al. 1995).

\subsection{Non-Darcy Flow Coefficient}

There is a great diversity in the correlations proposed in the literature for the expression of the non-Darcy flow coefficients (see Li and Engler 2001 for a review). This diversity can be attributed to the difference in lithology, in the pore geometry of the formation, and, therefore, in the flow patterns. This has motivated the use of many different parameters for the correlations reported in the literature. The major parameters considered are the permeability $(k)$, effective porosity $(\varepsilon)$, and tortuosity $(\tau)$, the three being related to each other (Li and Engler 2001).

One of the most commonly used correlations was obtained from experimental data and dimensional analysis by Geertsma (1974) for single-phase inertial flow in porous media:

$$
\beta=\frac{0.005}{k^{0.5} \varepsilon^{5.5}}
$$


Geertsma (1974) was also the first to propose an expression of the inertial coefficient for twophase flow, by replacing the permeability in Eq. 2 by the effective permeability $\left(K_{\alpha}=k k_{r \alpha}\right)$ and the porosity by the corresponding phase volume fraction $\left(\varepsilon S_{\alpha}\right), S_{\alpha}$ being the $\alpha$-phase saturation, thus giving

$$
\beta_{\alpha}=\frac{0.005}{K_{\alpha}^{0.5}\left(\varepsilon S_{\alpha}\right)^{5.5}} .
$$

More recently, the importance of including tortuosity $\tau$ in the correlation has been pointed out by Liu et al. (1995). In order to test Geertsma's proposition and to develop a general correlation for the non-Darcy flow coefficient, a large variety of single- and two-phase flow data were collected by these authors from various sources (Cornell and Katz 1953; Geertsma 1974; Evans et al. 1987; Evans and Evans 1988; Whitney 1988) including studies on consolidated and unconsolidated porous media as well as an analysis of the effect of an immobile liquid saturation. On the basis of this, Liu et al. (1995) proposed a more satisfactory correlation by including the tortuosity factor in the expression of the inertial coefficient given by

$$
\beta_{\alpha}=\frac{2.923 \times 10^{-6} \tau}{\varepsilon K_{\alpha}}
$$

with $\beta_{\alpha}$ and $K_{\alpha}$ expressed in SI units, the numerical constant having the unit of a length (m).

In the computations carried out in this study, the non-Darcy coefficient given in Eq. 4 was used for each phase with a tortuosity value of 1.9 (Wahyudi et al. 2000). Using the expression $K_{\alpha}=k k_{r \alpha}$ and identifying $\beta$ as $\frac{2.923 \times 10^{-6} \tau}{\varepsilon k}$ lead to

$$
\beta_{r \alpha}=k_{r \alpha}^{-1}
$$

This form corresponds to that suggested by many other authors (Lipinski 1982; Lee and Catton 1984; Saez and Carbonnell 1985). However, it must be noted that $\beta_{\alpha}$ is a user-fixed function, and its choice does not impose any restrictions on the numerical approach developed here. A more thorough discussion of these correlations is beyond the scope of this article.

\subsection{Reformulation of the Problem}

The Darcy-Forchheimer equation (Eq. 1) may be written in the form $(\alpha=o, w)$

$$
\mathbf{u}_{\alpha}=-\left[\frac{\mu_{\alpha}}{l}\left(l \mathbf{K}_{\alpha}^{-1}+\beta_{\alpha} R e_{\alpha}\right)\right]^{-1} \cdot\left(\nabla p_{\alpha}-\rho_{\alpha} \mathbf{g}\right)
$$

with the Reynolds number, $R e_{\alpha}$, associated with the $\alpha$-phase defined as

$$
R e_{\alpha}=\frac{\rho_{\alpha}\left\|\mathbf{u}_{\alpha}\right\| l}{\mu_{\alpha}}
$$

In this relationship, $l$ represents a characteristic length scale of the problem. In the literature, this length is generally associated with the pore scale and is typically taken equal to the size of the solid grains (Muskat 1937). In that case, this quantity can be estimated using relations between intrinsic permeability and porosity of the Kozeny-Carman type (Koederitz et al. 1989). A more suitable choice of this characteristic length scale allowing a better quantification of the inertial effects is proposed later on.

As suggested by Eq. 6, introducing a new expression of the mobility given by

$$
\mathbf{M}_{\alpha}=\left[\frac{\mu_{\alpha}}{l}\left(l \mathbf{K}_{\alpha}^{-1}+\beta_{\alpha} R e_{\alpha}\right)\right]^{-1}
$$


leads to a form of this equation similar to generalized Darcy's law. The latter is obtained if the term $\beta_{\alpha} R e_{\alpha}$ is negligible in comparison with $l \mathbf{K}_{\alpha}^{-1}$. An essential difference between the inertial problem we are dealing with and the classical Darcy's problem is the treatment of the nonlinearity associated with the special definition of the mobilities in our case. Indeed, in addition to the "classical" nonlinear dependence upon the saturation $\left(\mathbf{K}_{\alpha}\left(S_{w}\right), \beta_{\alpha}\left(S_{w}\right)\right.$, see below), mobilities are here functions of the Reynolds number, therefore, of the velocity leading to a non-linear momentum conservation equation in terms of velocity.

For our particular case of isotropic porous medium $\left(\mathbf{M}_{\alpha}=M_{\alpha} \mathbf{I}\right)$, the expression of the mobility given by Eq. 8 can be written as

$$
M_{\alpha}=\left[\frac{\mu_{\alpha}}{K_{\alpha}}\left(1+\frac{K_{\alpha} \beta_{\alpha}}{l} R e_{\alpha}\right)\right]^{-1} .
$$

This expression suggests that a relevant choice of the characteristic length is $l=k \beta$ giving the following expression of the Reynolds number:

$$
R e_{\alpha}=\frac{\rho_{\alpha}\left\|\mathbf{u}_{\alpha}\right\| k \beta}{\mu_{\alpha}}
$$

as proposed for a single-phase flow by Geertsma (1974). With this definition, the $\alpha$-phase mobility can be written as

$$
M_{\alpha}=\left[\frac{\mu_{\alpha}}{K_{\alpha}}\left(1+k_{r \alpha} \beta_{r \alpha} R e_{\alpha}\right)\right]^{-1} .
$$

In this manner, the local relative importance of inertial effects to the classical viscous ones can be predicted by a direct comparison of $k_{r \alpha} \beta_{r \alpha} R e_{\alpha}$ to 1 . Since $\beta_{r \alpha}=k_{r \alpha}^{-1}$ in our particular case, inertial effect can be directly appreciated by comparing $R e_{\alpha}$ to 1 . Moreover, this definition has the advantages of (i) involving macroscopic quantities $k$ and $\beta$ available from experiments, (ii) avoiding the introduction of a microscopic quantity (typically the grain size) that is most of the time unknown a priori and difficult to estimate precisely because of the absence of a universal relationship between macroscopic and microscopic characteristic lengths.

To the momentum conservation equations (Eq. 6), it is necessary to associate the mass conservation equations, unmodified in comparison to the Darcy regime, where the effective porosity, $\varepsilon$, is considered as a constant

$$
\varepsilon \frac{\partial S_{\alpha}}{\partial t}+\nabla \cdot \mathbf{u}_{\alpha}=0 \quad(\alpha=o, w)
$$

Closing the problem with the saturations and capillary pressure relationships leads to the following boundary value problem:

$$
\left\{\begin{array}{l}
\varepsilon \frac{\partial S_{\alpha}}{\partial t}+\nabla \cdot\left[-\mathbf{M}_{\alpha} \cdot\left(\nabla p_{\alpha}-\rho_{\alpha} \mathbf{g}\right)\right]=0 \quad(\alpha=o, w) \\
S_{w}+S_{o}=1 \\
p_{c}=p_{c}\left(S_{w}\right)
\end{array}\right.
$$

along with the required boundary and initial conditions.

In this study, capillary pressure and relative permeabilities are supposed to depend on $S^{*}$ only and are given by

$$
p_{c}\left(S_{w}\right)=p_{c_{0}}\left(1-S^{*}\right)^{\xi} \quad k_{r w}=\left(S^{*}\right)^{\gamma} \quad k_{r o}=\left(1-S^{*}\right)^{\theta}
$$

where $S^{*}$ is the reduced saturation defined as 


$$
S^{*}=\frac{S_{w}-S_{\mathrm{wi}}}{1-S_{\mathrm{wi}}-S_{\mathrm{or}}} .
$$

The saturations $S_{\mathrm{wi}}$ and $S_{\mathrm{or}}$ correspond to the irreducible wetting phase and residual nonwetting phase saturations, respectively. In Eq. $14 p_{c_{0}}$ represents the capillary pressure value when $S_{w}=S_{\text {wi }}$, whereas $\xi, \gamma$, and $\theta$ are constant exponents, the two latter being referred to as the Corey exponents (Corey 1954). It must be noted that the exact form of the relative permeability and capillary pressure functions, generally expressed as a function of $S_{w}$ and defined between the two end-points $\left(S_{\mathrm{wi}}\right.$ and $\left.1-S_{\mathrm{or}}\right)$, depends on the pore-space topology of the porous medium, its wetting properties, and fluids distributions (Marle 1972). They are generally obtained experimentally for each particular case (Corey 1954; Brooks and Corey 1966; Honarpour et al. 1986; Abdobal 2002). Special forms used in this study and generally admitted in the viscous-capillary regime are only a choice and do not impose any restrictions on the numerical development in which all these quantities are treated as nonlinear functions of the saturation.

Taking $S_{w}$ and $p_{o}$ as unknowns, Eq. 13 can be reformulated in terms of the total velocity $\mathbf{u}_{t}$, and the total mobility $\mathbf{M}$ defined as

$$
\begin{aligned}
& \mathbf{u}_{t}=\mathbf{u}_{w}+\mathbf{u}_{o} \\
& \mathbf{M}=\mathbf{M}_{w}+\mathbf{M}_{o} .
\end{aligned}
$$

This reformulation, based on incompressibility of the two fluids, has the advantage of simplifying the numerical scheme by introducing the divergence-free total velocity. Other formulations are proposed in the literature, namely, a formulation in parabolic form calling upon a simultaneous solution of the problem for the two pressures (or one saturation and one pressure; Aziz and Settari 1979) or one introducing a notion of global pressure (Chavent 1976). With our choice, the problem can, therefore, be written as

$$
\begin{gathered}
\nabla \cdot\left(-\mathbf{M} \cdot \nabla p_{o}+\mathbf{M}_{w} \cdot \nabla p_{c}+\left[\mathbf{M}_{w} \rho_{w}+\mathbf{M}_{o} \rho_{o}\right] \mathbf{g}\right)=0 \\
\varepsilon \frac{\partial S_{w}}{\partial t}+\nabla \cdot\left\{-\mathbf{M}_{w} \cdot\left[\nabla p_{o}-\nabla p_{c}-\rho_{w} \mathbf{g}\right]\right\}=0
\end{gathered}
$$

with initial and boundary conditions discussed below.

\subsection{Initial, Boundary, and Interface Conditions}

The initial oil-phase pressure and water-phase saturation are considered to be given in all the domains

$$
p_{o}(\mathbf{r}, t=0)=p_{0}(\mathbf{r}) ; \quad S_{w}(\mathbf{r}, t=0)=S_{0}(\mathbf{r}) .
$$

where $\mathbf{r}$ and $t$ are, respectively, the position vector and time variable.

Interface Conditions: Necessary boundary conditions include conditions at the interface, $\Gamma_{\omega \eta}$, between two different homogeneous regions $\omega$ and $\eta$ when considering a heterogeneous porous medium. At $\Gamma_{\omega \eta}$, flux and pressure continuity for each phase must be satisfied. These conditions can be written as

$$
\begin{aligned}
\mathbf{u}_{\alpha}^{\eta} \cdot \mathbf{n}_{\omega \eta} & =\mathbf{u}_{\alpha}^{\omega} \cdot \mathbf{n}_{\omega \eta} \text { at } \Gamma_{\omega \eta} \quad(\alpha=o, w) \text { i.e. } \mathbf{u}_{t}^{\eta} \cdot \mathbf{n}_{\omega \eta}=\mathbf{u}_{t}^{\omega} \cdot \mathbf{n}_{\omega \eta} \text { at } \Gamma_{\omega \eta} \\
p_{\alpha}^{\omega} & =p_{\alpha}^{\eta} \text { at } \Gamma_{\omega \eta} \quad(\alpha=o, w) \text { i.e. } p_{c}^{\omega}=p_{c}^{\eta} \text { at } \Gamma_{\omega \eta},
\end{aligned}
$$

where $\mathbf{n}_{\omega \eta}$ is the unit vector normal to $\Gamma_{\omega \eta}$ and directed from the $\omega$-region toward the $\eta$-region. Boundary Conditions: As classically used, conditions of the imposed pressure type, imposed flow rate type, or impermeable boundaries are considered. In the following, we use a domain 
delimited by an inlet, an outlet, and lateral surfaces on which the following boundary conditions are considered:

At the inlet: $\quad \mathbf{u}_{w} \cdot \mathbf{n}_{i}=u_{\text {imposed }}$ or $p_{o}=p_{\text {imposed }} ; \quad S_{w}=1-S_{\text {or }}$

On the lateral surfaces: $\mathbf{u}_{\alpha} \cdot \mathbf{n}_{l}=0 \quad(\alpha=o, w)$

At the outlet: $\left\{\begin{array}{c}p_{o}=p_{\text {atm }} ; \text { as long as } S_{w}\left(\mathbf{r}_{e}, t\right)<1-S_{\text {or }}, \mathbf{u}_{w} \cdot \mathbf{n}_{e}=0 \\ \text { When } S_{w}\left(\mathbf{r}_{e}, t\right)=1-S_{\text {or }} \text { simulation ends }\end{array}\right.$

Here, $\mathbf{r}_{e}$ is the position vector corresponding to the outlet face. We have denoted by $\mathbf{n}_{i}, \mathbf{n}_{e}$ and $\mathbf{n}_{l}$, respectively, the unit vectors normal to the inlet face, the outlet face, and the lateral surfaces, the latter being supposed impervious. Since our study focuses on imbibition, the inlet face is assumed to be in contact with water and therefore at a saturation of $1-S_{\text {or }}$. When flow rate is imposed, a suitable choice of a unique Reynolds number, $R e$, characteristic of the problem under consideration can be derived from Eq. 10 under the form

$$
R e=\max _{\kappa=\eta, \omega}\left(k_{\kappa} \beta_{\kappa}\right)\left\|\mathbf{u}_{t}\right\| \max _{\alpha=o, w}\left(\frac{\rho_{\alpha}}{\mu_{\alpha}}\right) .
$$

For an imposed pressure condition, the Reynolds number as defined above is time varying. For this case, an upper bound of the Reynolds number can be defined as follows:

$$
R e=\max _{\kappa, \alpha}\left(\frac{k_{\kappa} \beta_{\kappa} \rho_{\alpha}\left\|\mathbf{u}_{\alpha}^{\kappa}\right\|}{\mu_{\alpha}}\right) \quad(\alpha=o, w ; \kappa=\eta, \omega) .
$$

In this equation, an estimation of the velocity magnitude, $\left\|\mathbf{u}_{\alpha}^{\kappa}\right\|$, may be obtained on the basis of the Forchheimer equation for one-dimensional single-phase flow of the $\alpha$-phase in the $\kappa$-region in the absence of gravity. This estimation is given by

$$
\left\|\mathbf{u}_{\alpha}^{\kappa}\right\|=\frac{1}{2 \rho_{\alpha} \beta_{\kappa}}\left(-\frac{\mu_{\alpha}}{k_{\kappa}}+\sqrt{\frac{\mu_{\alpha}^{2}}{k_{\kappa}^{2}}+4 \rho_{\alpha} \beta_{\kappa} \frac{\triangle p_{\alpha}}{L}}\right) \quad(\alpha=o, w ; \kappa=\eta, \omega)
$$

in which $\triangle p_{\alpha}$ corresponds to the pressure difference between the inlet and outlet faces, i.e., over the total length of the medium $L$. With this estimation of the velocity, the Reynolds number can be written as

$$
R e=\frac{1}{2} \max _{\kappa, \alpha}\left(-1+\sqrt{1+\frac{4 \rho_{\alpha} \beta_{\kappa} k_{\kappa}^{2}}{\mu_{\alpha}^{2}} \frac{\triangle p_{\alpha}}{L}}\right) \quad(\alpha=o, w ; \kappa=\eta, \omega) .
$$

While the pressure boundary condition at the outlet face corresponds to the fact that oil leaves the porous medium at atmospheric pressure, the choice of the saturation boundary condition is rather delicate. The boundary condition proposed in Eq. 23 mimics a classical "end effect" (Marle 1972). Indeed, when simulating imbibition, the medium is initially saturated with oil, and water is injected. The fluid which, thus, primarily leaves the porous medium at the outlet face is oil. Water is accumulated at the outlet $\left(\mathbf{u}_{w} \cdot \mathbf{n}_{e}=0\right)$ as long as the saturation is less than $1-S_{\text {or }}$ and is allowed to break through only when the maximum water saturation is reached, which means that the front has entirely swept the medium. This boundary condition leads to exaggerated end effects under certain conditions. For this reason, an alternative outlet condition could also be considered. It consists in violating the capillary pressure relationship, not valid outside of the porous medium. Therefore, at the outlet face, water (and oil) can leave the medium without requiring any special condition on the saturation. 


\section{Discretization and Numerical Modeling}

The complete boundary value problem to be solved is given by Eqs. 18, 19, initial and boundary conditions (Eqs. 20, 23) as well as interfacial conditions (Eqs. 21, 22) required when the medium is heterogeneous.

Spatial discretization of this system of equations is performed using a finite volume method over a staggered grid and a first-order upwind scheme to estimate mobilities. The time scheme, similar to that used for two-phase flow in the Darcy regime, is of type IMPES in which oil pressure is determined implicitly and water saturation is calculated in an explicit manner (Aziz and Settari 1979; Chen et al. 2004). This scheme decouples pressure and saturation equations, thus reducing computational effort required for their solution. This method is efficient and simple and requires less computer memory compared to other methods such as simultaneous solution (SS) methods or fully implicit ones (sequential solution method SEQ; Aziz and Settari 1979). However, the IMPES method requires smaller time steps than implicit algorithms on the basis of a stability criterion associated with the explicit part of the scheme.

\subsection{Stability}

For two-phase flow in the Darcy regime, the IMPES scheme has two stability limits which can be studied independently (Aziz and Settari 1979). The first, rather complex, concerns the explicit treatment of the capillary pressure and depends on the magnitude of $\frac{\mathrm{d} p_{c}}{\mathrm{~d} s_{w}}$. The second is related to the explicit treatment of the mobilities and can be expressed in terms of a physical criterion stipulating that during a time step, each grid-block cannot be flooded by a volume of fluid larger than the pore volume it contains, i.e. Aziz and Settari (1979)

$$
\Delta t \leq \frac{\varepsilon \Delta x \Delta y \Delta z}{u_{t x} \Delta y \Delta z+u_{t y} \Delta z \Delta x+u_{t z} \Delta x \Delta y},
$$

where $\Delta x, \Delta y$, and $\Delta z$ are the grid sizes in the three directions of space and $u_{t x}, u_{t y}$, and $u_{t z}$ are the three components of the total velocity, $\mathbf{u}_{t}$. If the first criterion is ignored, the use of this second criterion, even in the Darcy regime, does not guarantee the overall stability of the IMPES method. In practice, the stability limit is often expressed in terms of a maximum allowable saturation variation in each grid-block during each time step (see Chen et al. 2004). In this study, without performing a detailed stability analysis, the criterion imposed by Eq. 28 was used with a suitable security coefficient less than 1 to ensure stability. A 3D numerical tool was developed to solve inertial two-phase flow in heterogeneous porous media on the basis of the physical model described above and with the features briefly detailed below.

\subsection{Treatment of Nonlinearities}

The oil pressure computation at time step $n+1$ makes use of $S_{w}$ evaluated at the previous time step $n$ as stipulated by the explicit scheme. However, another important issue is the choice of the node position for the estimation of the mobilities when evaluating velocities at the interface between two nodes. Indeed, the use of a staggered grid is such that velocities and, therefore, pressure gradients are evaluated at the interface between two grid-blocks, whereas saturations, and thus mobilities, are evaluated at the center of grid-blocks. In order to circumvent this difficulty, an upwind scheme classically used in petroleum engineering is employed to ensure physically meaningful results. It consists in estimating mobilities using the value of saturation on the node located upwind to the flow with regard to the interface. 
As mentioned earlier, the main additional difficulty lies in the correct estimation of the velocity-dependent part of the mobilities. For this reason, special care must be taken in treating these terms. Three different methods were considered: the fixed point iteration method, the purely explicit scheme, or a two-step Adams-Bashford method. The latter features instabilities behind the saturation front as observed for the classical Darcy case unless very small grid sizes and time steps are used (Aziz and Settari 1979). Several tests performed on homogeneous media indicate that the most satisfactory results considering both the rarefaction zone behind the front and the front itself are obtained by the fixed point iteration method. Although this conclusion needs to be examined more thoroughly before generalizing to a heterogeneous medium, this method was adopted for all the simulations performed in this article and is briefly described below.

The fixed point method is based on an iterative process on velocities to compute the oil pressure $p_{o}$. This iterative process starts with velocities of each phase initialized with those obtained at the previous time step ( 0 for the first time step). Mobilities are estimated from these velocity fields and are used to solve for the oil pressure to calculate a new value of the flow velocity field (first iteration). Fixed point iterations are repeated, without any time iteration, until the relative difference between the norm of the velocity of each phase at any grid point for two successive iterations is less than a user-defined criterion.

\subsection{Algorithm}

On the basis of the initial imposed saturation field, relative permeabilities, capillary pressure, and relative inertial coefficients are calculated at each point at the initial time $t=0$. As discussed earlier, these parameters are considered as given data for our simulations. This allows the computation of the oil pressure at each node at $t=\Delta t$ in an implicit manner by solving the discretized version of Eq. 18. During this resolution step, saturations in the capillary pressure and in the mobilities are taken at the previous time step (initial condition for the first time step). The symmetric linear system corresponding to the implicit treatment of the oil pressure is solved by a conjugate gradient method. Using Eq. 19, the water saturation at the same time step is computed in an explicit manner. Then, $p_{w}, \mathbf{u}_{o}$, and $\mathbf{u}_{w}$, and, therefore, the total velocity, $\mathbf{u}_{t}$, are computed. Before proceeding to the next time step, the corresponding $\Delta t$ is determined based on Eq. 28.

\section{Validation}

Validation of our numerical model is performed in comparison with a semi-analytical solution of "Buckley-Leverett" type in a special case. In fact, the classical semi-analytical solution of Buckley and Leverett (1942) for one-dimensional flow of two incompressible, immiscible fluids in homogeneous porous media under negligible capillary pressure assumption and in the Darcy regime can be further extended to the case of inertial flow described by the generalized Darcy-Forchheimer model.

\subsection{Analytical Solution for Inertial Flow}

In the case of one-dimensional inertial flow in the $x$ direction in a semi-infinite homogeneous porous medium of constant cross-section $A$, the mass and momentum conservation equations presented above (Eqs. 1, 12) can be written as follows $(\alpha=o, w)$ : 


$$
\begin{aligned}
-\frac{\partial u_{\alpha}}{\partial x} & =\varepsilon \frac{\partial S_{\alpha}}{\partial t} \\
-\frac{\partial p_{\alpha}}{\partial x} & =\frac{\mu_{\alpha}}{K_{\alpha}} u_{\alpha}+\beta_{\alpha} \rho_{\alpha}\left|u_{\alpha}\right| u_{\alpha},
\end{aligned}
$$

where $u_{\alpha}$ is the Darcy velocity for the $\alpha$-phase. In order to complete the description of the problem, initial and boundary conditions must be indicated:

$$
S_{w}(x, t=0)=S_{\mathrm{wi}} ; \quad u_{o}(x=0, t)=0 ; \quad u_{w}(x=0, t)=q / A .
$$

The last condition corresponds to the continuous injection of water at a known constant flow rate, $q$, at the inlet face of the porous medium $(x=0)$.

Following the work of $\mathrm{Wu}$ (2001), the concept of fractional flow is employed to simplify the governing equation (29) in terms of saturations only. The fractional flow of the $\alpha$-phase is defined as

$$
f_{\alpha}=\frac{u_{\alpha}}{u_{t}} \quad(\alpha=o, w)
$$

with the evident relationship:

$$
f_{w}+f_{o}=1
$$

Introduction of $f_{w}$ and $f_{o}$ in the momentum balance equations and use of the zero capillary pressure assumption lead to the following analytical expression of the fractional flow for the water-phase:

$$
f_{w}=\frac{\left[-a+\left(a^{2}+4 u_{t} b\left(\frac{\mu_{o}}{K_{o}}+\rho_{o} \beta_{o} u_{t}\right)\right)^{1 / 2}\right]}{2 b u_{t}}
$$

where

$$
a=\frac{\mu_{o}}{K_{o}}+\frac{\mu_{w}}{K_{w}}+2 \rho_{o} \beta_{o} u_{t} ; \quad b=\rho_{w} \beta_{w}-\rho_{o} \beta_{o}
$$

Since $\mathrm{K}_{\alpha}$ and $\beta_{\alpha}(\alpha=w, o)$ only depend on water saturation, Eq. 34 indicates that, for a given injection rate-therefore, a constant total velocity $u_{t}$ - and for given fluid properties, $f_{w}$ is a function of water saturation only. The mass balance equation (Eq. 29) can, hence, be written in terms of the fractional flow:

$$
\frac{\partial S_{w}}{\partial t}+W\left(S_{w}\right) \frac{\partial S_{w}}{\partial x}=0
$$

where

$$
W\left(S_{w}\right)=\frac{d x}{d t}=\frac{q}{\varepsilon A}\left(\frac{d f_{w}}{d S_{w}}\right)
$$

Equation 36 is the frontal equation for non-Darcy immiscible two-phase displacement and has the same form as the classical Buckley-Leverett equation. The expression (37) shows that, for a given time and injection flow rate, each value of the saturation, $S_{w}$, is propagated in homogeneous porous media at a constant velocity $W\left(S_{w}\right)$. Actually, Eq. 36 is a hyperbolic transport equation which is classically solved using the method of characteristics. The Welge tangent method (Welge 1952) is used to determine a constant front (or shock) saturation, $S_{f}$, ahead of a smoothly varying saturation (Marle 1972) corresponding to the rarefaction zone. This is required to keep a physically meaningful saturation profile. 
Table 1 Numerical data for one-dimensional flow in homogeneous porous media

\begin{tabular}{llllllllllll}
\hline $\begin{array}{l}k \\
\left(\mathrm{~m}^{2}\right)\end{array}$ & $k_{r w}$ & $k_{\text {ro }}$ & $\varepsilon$ & $\tau$ & $\begin{array}{l}\beta \\
\left(\mathrm{m}^{-1}\right)\end{array}$ & $\beta_{r \alpha}$ & $S_{\mathrm{wi}}, S_{\mathrm{or}}$ & $\begin{array}{l}\rho_{w} \\
\left(\mathrm{~kg} \mathrm{~m}^{-3}\right)\end{array}$ & $\begin{array}{l}\rho_{o} \\
\left(\mathrm{~kg} \mathrm{~m}^{-3}\right)\end{array}$ & $\begin{array}{l}\mu_{w} \\
(\mathrm{Pas})\end{array}$ & $\begin{array}{l}\mu_{o} \\
(\mathrm{Pas})\end{array}$ \\
\hline $10^{-11}$ & $S^{* 2}$ & $\left(1-S^{*}\right)^{2}$ & 0.4 & 1.9 & $1.39 \times 10^{6}$ & $k_{r \alpha}^{-1}$ & 0.1 & 1,000 & 900 & $10^{-3}$ & $5 \times 10^{-3}$ \\
\hline
\end{tabular}

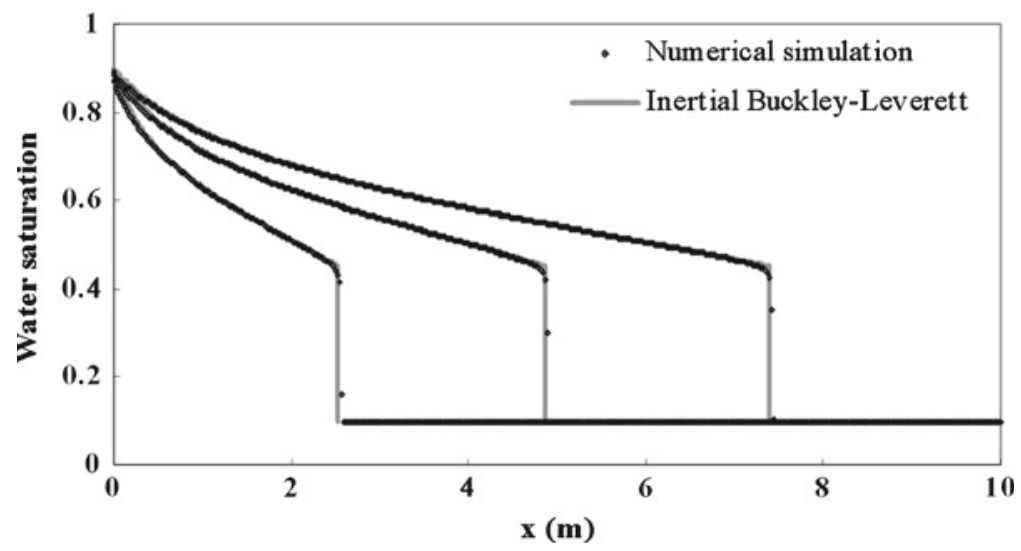

Fig. 1 Comparison of saturation profiles for various times $(t=10.4 \mathrm{~s}, 20 \mathrm{~s}$, and $30.4 \mathrm{~s})$ obtained by the numerical code with those resulting from the semi-analytical resolution of the Buckley-Leverett type; number of grid-blocks $N=400, \Delta t=0.08 \mathrm{~s}$

\subsection{Comparison with Numerical Simulations_-Validation}

In this section, the system of equations is solved numerically for a $1 \mathrm{D}$ homogeneous porous medium supposing negligible capillary effects. Results are compared to those obtained analytically using the Buckley-Leverett approach presented in the previous paragraph. This comparison was carried out with the physical parameters of Table 1 and the effective and relative inertial coefficients, $\beta_{\alpha}$ and $\beta_{r \alpha}$ respectively, given in Eqs. 4 and 5. For the case considered in this section, $u_{t}$ and $R e$ are respectively equal to $5 \times 10^{-2} \mathrm{~m} \mathrm{~s}^{-1}$ and 0.69 (see Eq. 24). A more detailed discussion of the importance of inertial effects versus Reynolds number is proposed in the next section.

The evolution of the water saturation profile, $S_{w}(x, t)$, in the medium obtained by direct simulation on the one hand (using a one-dimensional version of the code with imposed flow rate at the inlet face) and by the quasi-analytical resolution of the Buckley-Leverett type of equation on the other hand shows a very good agreement as indicated in Fig. 1. This result validates our numerical algorithm in the particular case of one-dimensional two-phase inertial flow in homogeneous porous media without capillary pressure.

\section{Numerical Experiments}

In this section, we present numerical simulations performed in different situations, including homogeneous and heterogeneous media with capillary pressure. The objective is to highlight, from comparison with results obtained with the classical Darcy model, the impact of inertial terms on the structure and kinetics of the saturation profiles. 
Accuracy of simulation results has been controlled through the estimation of relative errors on the mass balance of each phase. The relative error on the cumulated mass balance (with respect to the pore volume) for each phase (water or oil) is less than $10^{-8}$ at any time during the whole simulation for our $1 \mathrm{D}$ experiments and less than $10^{-5}$ for the $2 \mathrm{D}$ ones. The largest relative errors for the $2 \mathrm{D}$ case correspond to cases with spikes at the interface between the two regions due to the particular choice of capillary pressure contrasts in this study (see results of the numerical experiments).

\subsection{Comparison of Darcy and Non-Darcy (Forchheimer) Flow Regimes}

In this paragraph, we highlight the influence of the inertial terms on the saturation profiles for different Reynolds numbers, $R e$, defined in Eq. 24 in the case of 1D flows in homogeneous porous media. For a given $R e$, saturation profiles are obtained by solving the boundary value problem corresponding to two-phase flow in the Darcy regime. These profiles are compared to those obtained by the resolution of the boundary value problem (Eqs. 18-23) taking into account the additional Forchheimer term corresponding to inertial effects. In these simulations, the flow rate is imposed at the inlet face. Saturation profiles are presented at two different times (see Fig. 2). Three different inlet velocities $u_{w}(x=0)=u_{t}=10^{-3}, 10^{-2}$, and $10^{-1} \mathrm{~m} \mathrm{~s}^{-1}$ corresponding to Reynolds number, respectively, equal to $0.014,0.14$, and 1.4 were considered. Although using Re defined by Eq. 24 allows a discussion of the inertial effects by a simple comparison of this number with unity, we also indicate the corresponding values of a "classical Reynolds number" defined as $R e_{\mathrm{cl}}=\max _{\alpha}\left(\rho_{\alpha} / \mu_{\alpha}\right)\left\|\mathbf{u}_{t}\right\| d$ for completeness. As mentioned earlier, $d$ is the grain size obtained from the Kozeny-Carman relationship (Koederitz et al. 1989):

$$
d=(1-\varepsilon)\left(150 k / \varepsilon^{3}\right)^{0.5}
$$

giving a value of $d=10^{-4} \mathrm{~m}$ here. The corresponding values of $R e_{\mathrm{cl}}$ for the three cases under consideration are, respectively, $0.1,1$, and 10 . The homogeneous medium for which the properties are reported in Table 1 is $10 \mathrm{~m}$ long, and its capillary pressure is taken as $p_{c}\left(S_{w}\right)=500\left(1-S^{*}\right)^{2}$ in SI units. While inertial effects remain negligible for $R e$ roughly below 0.014 , they become significant for a $R e$ of 0.14 and beyond this value. As expected, due to additional friction, the displacement of the saturation front is inhibited by inertial effects. Ignoring these effects, for Reynolds numbers roughly larger than 0.1 , may, therefore, lead to significant errors in watercut curves and a noticeable underestimation of the breakthrough time.

\subsection{Heterogeneous Porous Media}

In this part of the study, results of simulations carried out on heterogeneous media comprising two regions ( $\eta$ and $\omega$ ) in one- and two-dimensional configurations depicted in Fig. 3 are presented.

\subsubsection{One-Dimensional Configurations}

The configuration represented in Fig. 3a is used with different values of the injection flow rate (Table 2) leading to different Reynolds numbers. The flow is perpendicular to two layers each of length $5 \mathrm{~m}$. Capillary pressure and relative permeability curves correspond to relations given in Eq. 14 with the parameters indicated in Table 3. Moreover, $\beta_{r \alpha}, \rho_{\alpha}$, and $\mu_{\alpha}$ were taken equal to those given in Table 1 . 


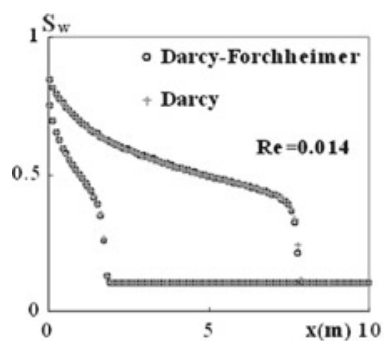

(a)

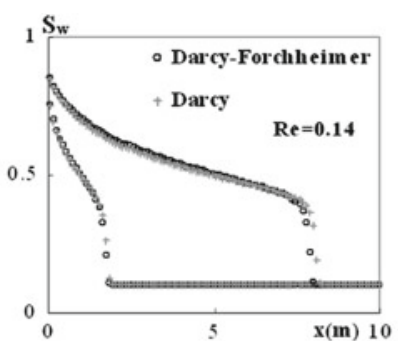

(b)

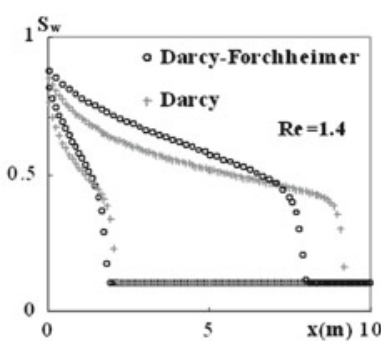

(c)

Fig. 2 Water saturation profiles along the medium for three cases from left to right: a $t=300$ and $1,400 \mathrm{~s}$ for a Reynolds number of $0.014, \mathbf{b} t=30 \mathrm{~s}$ and $145 \mathrm{~s}$ for a Reynolds number of $0.14, \mathbf{c} t=3.5 \mathrm{~s}$ and $16.5 \mathrm{~s}$ for a Reynolds number of $1.4, N=100$

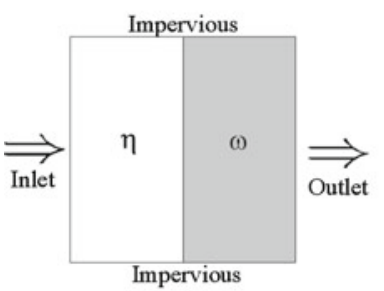

(a)

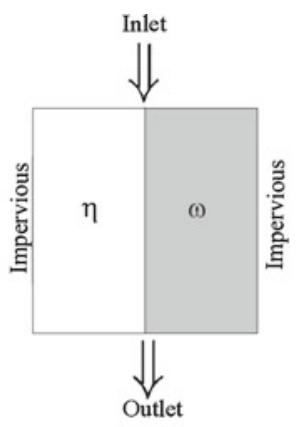

(b)

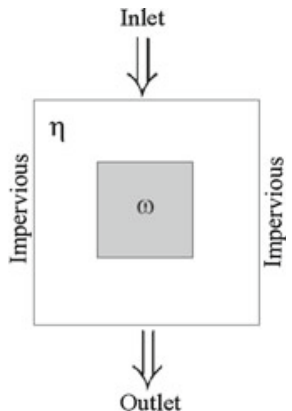

(c)

Fig. 3 Geometrical configurations for heterogeneous porous media considered in the simulations. a 1D layered heterogeneous medium (flow is parallel to the layers), b 2D layered heterogeneous medium (flow is perpendicular to the layers), C 2D nodular heterogeneous medium

Table 2 Different injection rates and the corresponding Reynolds and capillary numbers for 1D heterogeneous simulations

\begin{tabular}{lllll}
\hline Injection rate & $10^{-6} \mathrm{~m} \mathrm{~s}^{-1}$ & $10^{-4} \mathrm{~m} \mathrm{~s}^{-1}$ & $7 \times 10^{-3} \mathrm{~m} \mathrm{~s}^{-1}$ & $10^{-1} \mathrm{~m} \mathrm{~s}^{-1}$ \\
\hline Reynolds number & $2.46 \times 10^{-5}$ & $2.46 \times 10^{-3}$ & $1.73 \times 10^{-1}$ & 2.46 \\
Capillary number $(\eta)$ & 40 & 0.4 & $5.7 \times 10^{-3}$ & $4 \times 10^{-4}$ \\
Capillary number $(\omega)$ & 9 & 0.09 & $1.29 \times 10^{-3}$ & $9 \times 10^{-5}$ \\
\hline
\end{tabular}

Two test cases consisting in injecting water into the more permeable zone $(\eta)$ and the less permeable zone $(\omega)$, respectively, are analyzed. First, for a very small Reynolds number of $2.46 \times 10^{-5}$, the saturation profiles (Fig. 4) show similar behavior as those reported in the literature (Chang and Yortsos 1989; Dale et al. 1997) for cases in the Darcy regime. Indeed, a downward peak is observed when liquid flows from a more permeable zone to a less permeable one ( $\eta \omega$ interface), and the contrary happens when the order is reversed ( $\omega \eta$ interface). This behavior is more pronounced at lower Reynolds numbers and is a consequence of the capillary dominance of the flow, which can be quantified through a capillary number

$$
C a_{\kappa}=\frac{\sigma \sqrt{k_{\kappa} \varepsilon_{\kappa}}}{L \mu_{o} u_{t}} \quad(\kappa=\eta, \omega)
$$




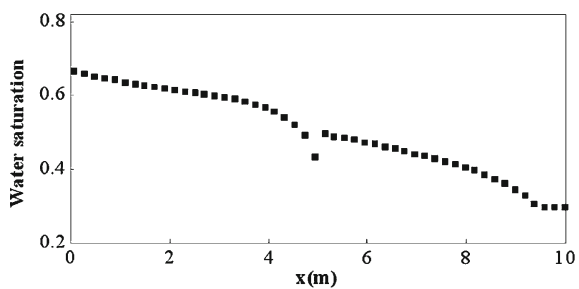

(a)

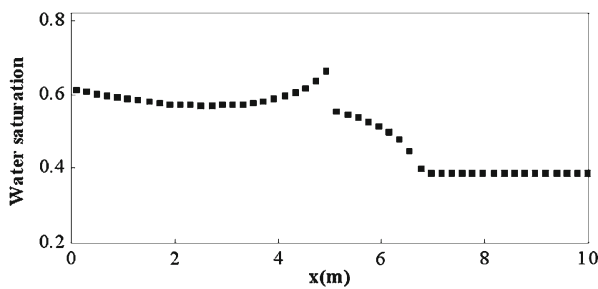

(b)

Fig. 4 Saturation fields for a injection into the more permeable zone $\eta\left(t=5.86 \times 10^{5} \mathrm{~s}\right)$ and $\mathbf{b}$ injection into the less permeable zone $\omega\left(t=4.24 \times 10^{5} \mathrm{~s}\right)$

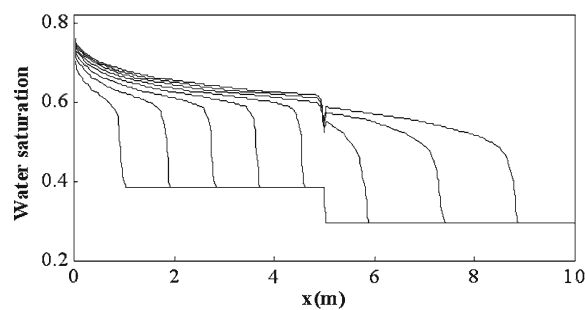

(a)

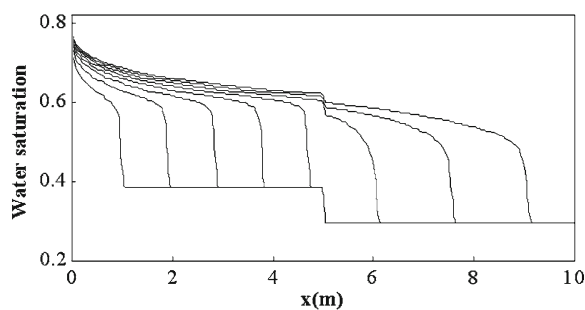

(b)

Fig. 5 Saturation profiles along the medium for various times for one-dimensional flow in a heterogeneous porous medium (flow rate imposed at the inlet face in the more permeable region $\eta$ ), $N=200$. a $R e=$ $2.46 \times 10^{-3}$ (Darcy regime) for $t=10^{3}, 2 \times 10^{3}, 3 \times 10^{3}, 4 \times 10^{3}, 5 \times 10^{3}, 6 \times 10^{3}, 7 \times 10^{3}$ and $8 \times 10^{3} \mathrm{~s}$, b $R e=0.17$ for $t=15,30,45,60,75,90,105$ and $120 \mathrm{~s}$

where $\sigma$ is the interfacial tension taken equal to $30 \times 10^{-3} \mathrm{Nm}^{-1}$, and $L$ is the length of the sample. Although there are different possible definitions of the capillary number, our discussion is not altered by this choice. Here, in Eq. 39 inspired by the definition proposed by Chang and Yortsos (1989) or Dale et al. (1997), higher $C a$ corresponds to the dominance of capillary effects. While cases with $R e=2.46 \times 10^{-3}$ can be considered as an intermediate case, for the two higher $R e$, capillary effects are definitely negligible (Table 2 ). These two cases correspond to flows in the inertial regime and those with lower Re correspond to Darcy regime flows.

For a Reynolds number equal to 0.17 (Fig. 5b), the form of the saturation profiles are only slightly modified in comparison to those with $R e=2.46 \times 10^{-3}$ corresponding to the Darcy regime (Fig. 5a). In this range of relatively small $R e$, the behavior observed at the $\eta \omega$ interface is due to capillary effects as mentioned above in addition to the contrast on $k, k_{r}, S_{\mathrm{wi}}, S_{\mathrm{or}}$, and $\varepsilon$. This behavior disappears at higher $R e$. In fact, further increase of the flow rate leads to significant modifications in the structure of the saturation profiles (Fig. 6). In particular, it can be seen in this last case that the sign of the saturation jump at the $\eta \omega$ interface is reversed when compared to the saturation profiles obtained at lower $R e$ and reported in Fig. 5. As indicated earlier, this behavior results from inertial effects only and is not due to capillary forces. Indeed, simulations performed with and without capillary pressure lead to similar results indicating that capillary effects are not responsible for the observed behavior. As expected, oil is expelled from the medium in a more efficient manner for higher Reynolds numbers $(R e=2.46)$. For this case, a numerical simulation using the Darcy model would lead to very different and, therefore, erroneous results (Fig. 6). 


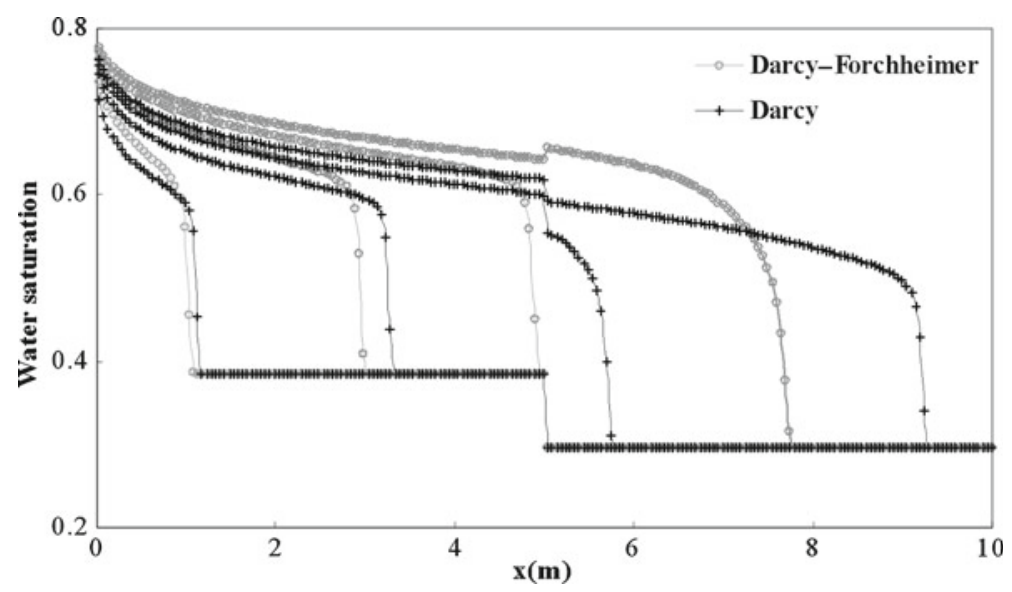

Fig. 6 Saturation profiles along the medium for various times (1.2, 3.6, 6, and 8.4 s) for one-dimensional flow in a heterogeneous porous medium (flow rate imposed at the inlet face in the more permeable region $\eta$ ); Reynolds number: $R e=2.46$. Results using the Darcy model are compared to those using the Darcy-Forchheimer model

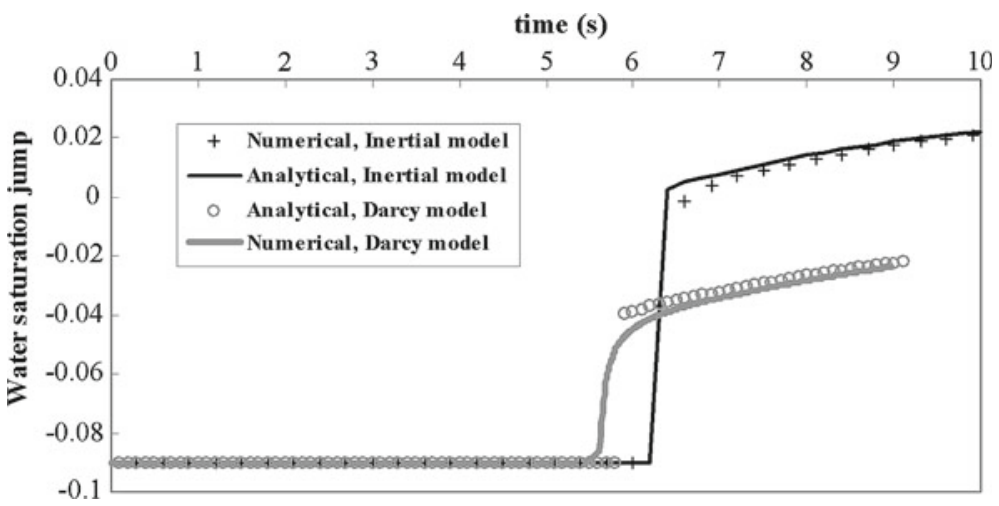

Fig. 7 The saturation jump $S w_{\eta}-S w_{\omega}$ at the interface $\eta \omega$ as a function of time: comparison between analytical and numerical results using inertial and Darcy models

For $R e=2.46$, since capillary effects are negligible, saturation jump at the $\eta \omega$ interface can be obtained semi-analytically. In fact, the "inertial Buckley-Leverett" formulation described above can be used in the first region to determine semi-analytically the saturation at any time on the left-hand side of the permeability discontinuity. Using the fact that the fractional flow must be continuous at this discontinuity, the saturation can be calculated on the right-hand side of the discontinuity by inverting the fractional flow function in this second region, thus providing the saturation jump at the discontinuity. The saturation jump obtained numerically is in good agreement with semi-analytical results (Fig. 7) confirming again the validity of our numerical solution. Moreover, using the Darcy model for this case leads to a negative saturation jump at the interface, whereas the Darcy-Forchheimer model gives positive saturation jumps once the front has crossed the interface (Figs. 6, 7). This is a result of the modified fractional flow function (Eq. 34) as compared to the classical Darcy regime fractional flow given by $f_{w}=\frac{\mu_{o} / K_{o}}{\left(\mu_{w} / K_{w}\right)+\left(\mu_{o} / K_{o}\right)}$. 


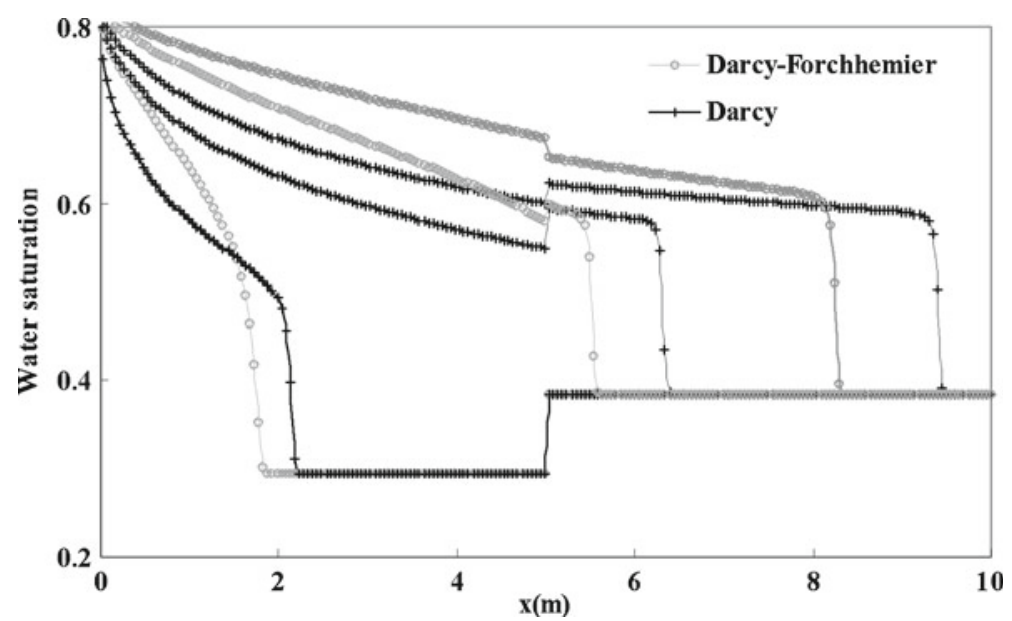

Fig. 8 Saturation profiles along the medium for various times (1.2, 3.6, and 8.4 s) for one-dimensional flow in a heterogeneous porous medium (flow rate imposed at the inlet face); water injected into the less permeable region $\omega$; Reynolds number: $R e=2.46$. Results using the Darcy model are compared to those using the Darcy-Forchheimer model, $N=200$

In order to confirm this observation, water has been injected into the low permeability zone $(\omega)$ for a high Reynolds number $(R e=2.46)$. The corresponding water-saturation profiles are illustrated in Fig. 8. There is a significant difference between the saturation fields obtained using the Darcy-Forchheimer model and those obtained using the Darcy model. In fact, in addition to the global time-lag inherent to the Darcy-Forchheimer model, the saturation jump at the $\omega \eta$ interface exhibits a reversed behavior with respect to the Darcy model.

For all the cases, a strong saturation change at the interface between the two regions is obtained. At low Reynolds number, it is a saturation jump related to the pressure continuity for both phases and, therefore, capillary pressure continuity at the interface between the two regions in addition to fluxes continuity in each phase. However, at higher Reynolds numbers, when inertial effects dominate, the saturation change is a consequence of the contrast between the properties of the two regions $\left(k, k_{r \alpha}, \varepsilon, S_{\mathrm{wi}}, S_{\mathrm{or}}, \beta, \beta_{r \alpha}\right)$. This behavior observed in the classical Darcy regime persists, but can be reversed when inertia is present.

\subsubsection{Two-Dimensional Configurations}

In this paragraph, the results of simulations carried out on heterogeneous media comprising two regions ( $\eta$ and $\omega$ ) in two-dimensional configurations are presented. These simulations are performed with the parameters of Table 3 . The values of $\beta_{r \alpha}, \rho_{\alpha}$, and $\mu_{\alpha}$ are taken equal to those given in Table 1. Two cases are considered:

- Stratified case corresponding to a two-layer medium with flow parallel to the layers of thickness $0.5 \mathrm{~m}$ and of length $1 \mathrm{~m}$ (Fig. 3b).

- Nodular case corresponding to a heterogeneous medium composed of a block of $\eta$-region with dimensions $1 \mathrm{~m} \times 1 \mathrm{~m}$, with a centered inclusion of $\omega$-region of size $0.25 \mathrm{~m} \times 0.25 \mathrm{~m}$ (Fig. 3c).

For cases studied here, pressure is imposed at the inlet face. The Reynolds number provided below is, therefore, given by Eq. 27. Moreover, for all cases, $40 \times 40$ grid blocks have been considered. 
Table 3 Numerical data for the two regions for simulations of inertial flow in heterogeneous porous media

\begin{tabular}{llllllllll}
\hline & $k\left(\mathrm{~m}^{2}\right)$ & $\beta\left(\mathrm{m}^{-1}\right)$ & $\gamma$ & $\theta$ & $\varepsilon$ & $S_{\mathrm{wi}}$ & $S_{\mathrm{or}}$ & $p_{c 0}(\mathrm{~Pa})$ & $\xi$ \\
\hline$\eta$-Region & $10^{-10}$ & $1.274 \times 10^{5}$ & 3 & 3 & 0.436 & 0.385 & 0.185 & 500 & 2 \\
$\omega$-Region & $10^{-11}$ & $2.468 \times 10^{6}$ & 2 & 2 & 0.225 & 0.295 & 0.178 & 5,000 & 2 \\
\hline
\end{tabular}

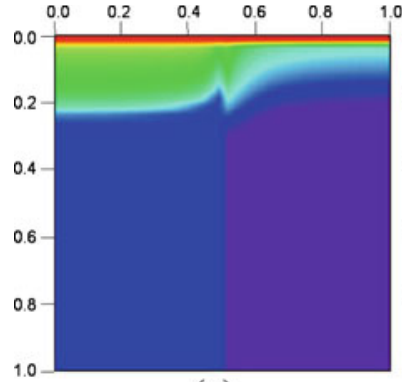

(a)

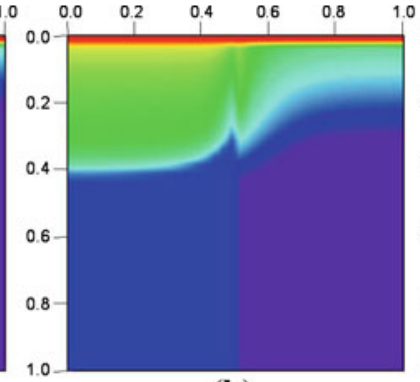

(b)

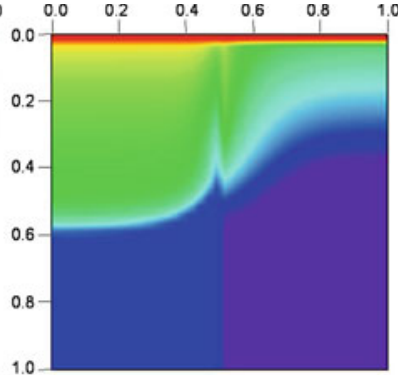

(c)

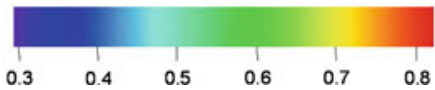

Fig. 9 Evolution of the saturation fields at three given times: a $t=200 \mathrm{~s}$, $\mathbf{b} t=400 \mathrm{~s}$, c $t=600 \mathrm{~s}$ for two-dimensional flow in a two-layer medium. The Darcy-Forchheimer model is used with imposed pressure at the inlet, $R e=6.3 \times 10^{-3}$

Stratified Case: Saturation fields obtained for a low Reynolds number $\left(R e=6.3 \times 10^{-3}\right)$ are first presented (Fig. 9). Pressures imposed at the inlet and outlet faces are respectively $1.05 \times 10^{5}$ and $10^{5} \mathrm{~Pa}$. In this particular case of low pressure gradient in the medium, contrasts of the capillary pressure and capillary pressure gradient in the two layers lead to a perturbed saturation profile at the interface between these two layers. This effect was reproduced with finer grids and smaller time steps highlighting the signature of a physical mechanism. In fact, this can be explained by a transverse capillary suction from the more permeable $\eta$-region featuring low capillary effects towards the $\omega$-region where capillary effects are ten times larger. Since the longitudinal flow rate is small, the saturation profile is strongly affected by this capillary cross flow. To be convinced of that, simulations with smaller capillary pressure contrasts were performed leading to smooth saturation fronts.

For larger values of the Reynolds number, capillary effects become insignificant in comparison to inertial and viscous effects and simulations with and without capillary pressure lead to identical results. For instance, for a larger value of the Reynolds number $(R e=3.1)$, corresponding to imposed inlet and outlet pressures of $10^{7}$ and $10^{5} \mathrm{~Pa}$ respectively, saturation profiles (reported in Fig. 10) exhibit a saturation front in each layer with a sharp but monotonic saturation change.

Finally, for a Reynolds number equal to 3.1, saturation fields obtained using the DarcyForchheimer model presented in Fig. 10 are compared to those obtained for the same times using the Darcy model (Fig. 11). The latter show a much faster propagation of the front in the more permeable $\eta$-region (at $t=0.44 \mathrm{~s}$, the difference in the location of the saturation front is about $29 \mathrm{~cm}$ ), while there is practically no difference in the front displacement in the less permeable $\omega$-region (front locations only differ about $1 \mathrm{~cm}$ at the same time). We note here that the Reynolds number as defined in Eq. 27 is mainly representative of the behavior 


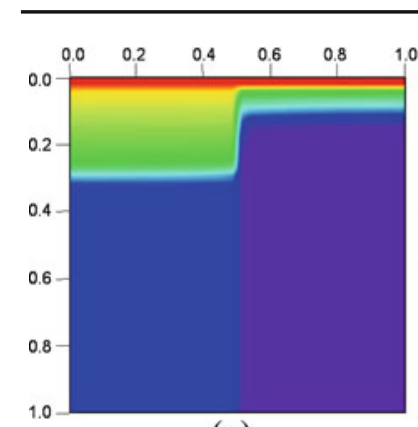

(a)

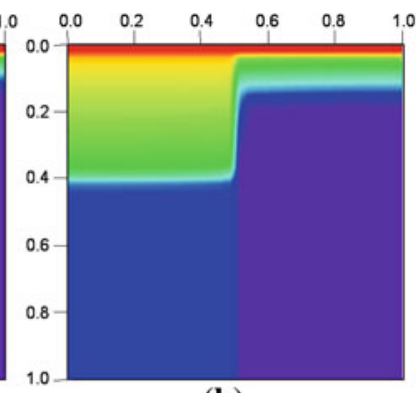

(b)

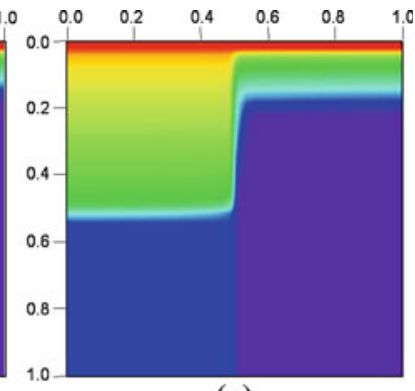

(c)

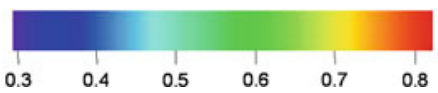

Fig. 10 Evolution of the saturation fields at three given times: a $t=0.22 \mathrm{~s}, \mathbf{b} t=0.33 \mathrm{~s}, \mathbf{c} t=0.44 \mathrm{~s}$ for two-dimensional flow in a two-layer medium. The Darcy-Forchheimer model is used with imposed pressure at the inlet, $R e=3.1$.

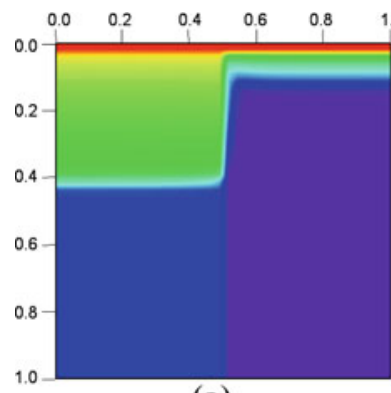

(a)

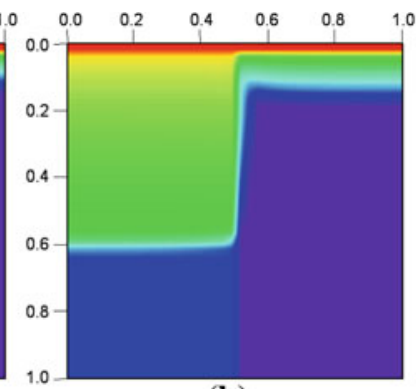

(b)

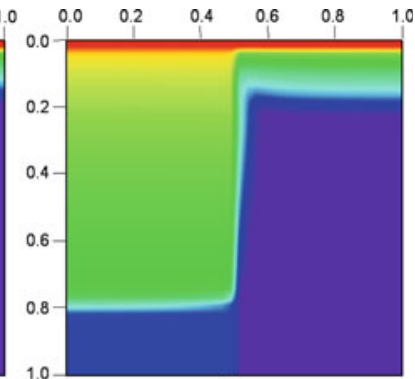

(c)

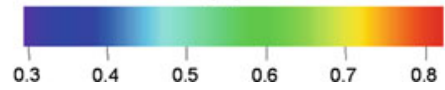

Fig. 11 Evolution of the saturation fields at three given times: $\mathbf{a} t=0.22 \mathrm{~s}, \mathbf{b} t=0.33 \mathrm{~s}$, c $t=0.44 \mathrm{~s}$ for two-dimensional flow in a two-layer medium. Same case as in Fig. 10 using the Darcy model

in the more permeable zone, since the maximum value in this relation is obtained for the water-phase $(\alpha=w)$ in the $\eta$-region.

Nodular case: For a small Reynolds number $\left(\operatorname{Re}=6.3 \times 10^{-3}\right)$, corresponding to imposed inlet and outlet pressures of $1.05 \times 10^{5}$ and $10^{5} \mathrm{~Pa}$, respectively, in the case of an inclusion of a less permeable zone in a more permeable one, capillary effects have significant influence on the saturation fields (see Fig. 12). As already discussed for the stratified case, our choice of capillary pressure curves leads to a perturbed saturation front at the interface between the two media. This is the result, already described for the stratified medium, of capillary-induced cross flow at the interface between the two regions.

This is evidenced from Fig. 13 where the velocity vector map in the water-phase is superimposed on the saturation field of Fig. 12b. Capillary suction is particularly visible at the upper corners of the inclusion having the largest capillary pressure. This leads to a trapped oil zone in the upper central part of this inclusion remaining at later times (see Fig. 12c). Similar behavior has been observed in the literature (Virnovsky et al. 2004) although in a somewhat different context since they consider a difference in the wettability of the two rocks (the capillary pressure curve for one of the rocks is negative over a large range of saturations). 


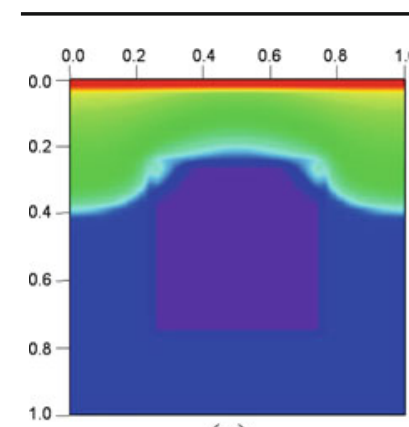

(a)

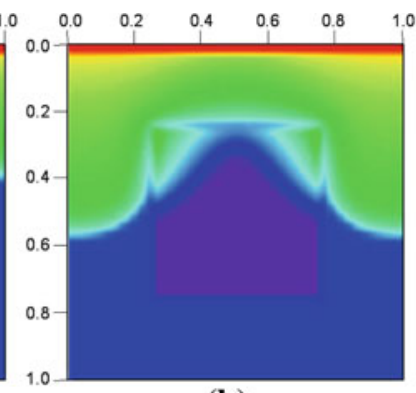

(b)

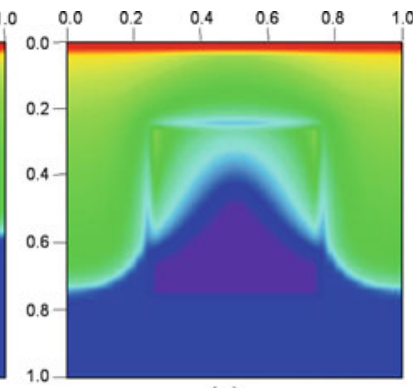

(c)

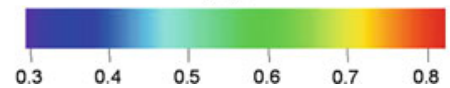

Fig. 12 Evolution of the saturation fields at three given times: a $t=400 \mathrm{~s}, \mathbf{b} t=600 \mathrm{~s}$, c $t=800 \mathrm{~s}$ for two-dimensional flow in a nodular medium with $R e=6.3 \times 10^{-3}$ : simulation with capillary pressure and imposed pressure at the inlet

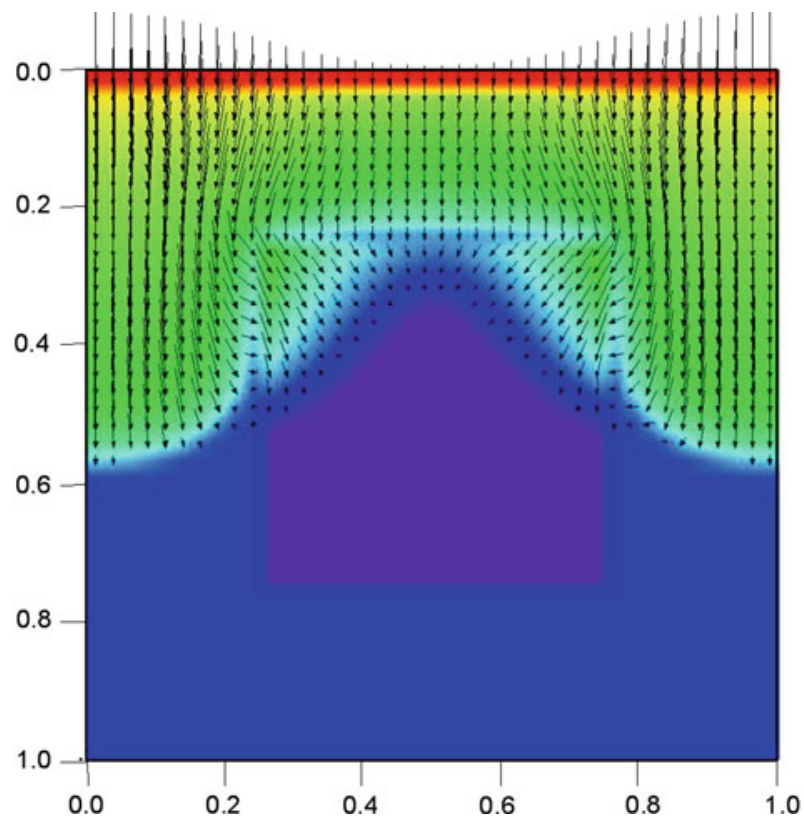

Fig. 13 Velocity vector map in the water phase superimposed on the saturation field of Fig.12b $(t=600 \mathrm{~s})$

For larger Reynolds numbers (Fig. 14), capillary effects are negligible since a simulation without capillary pressure leads to strictly similar results.

The last comparison is performed between saturation fields obtained using the DarcyForchheimer model (Fig. 14), and those obtained using the Darcy model (Fig. 15) for a Reynolds number equal to 3.1. Again, for a given time, the saturation front has propagated much further in the more permeable matrix when the Darcy model is used (overestimation of the saturation front by about $28 \mathrm{~cm}$ for $t=0.44 \mathrm{~s}$ in the more permeable region), whereas there is no significant difference in the saturation field in the less permeable inclusion (difference of $1 \mathrm{~cm}$ only in the saturation front at the same time). 


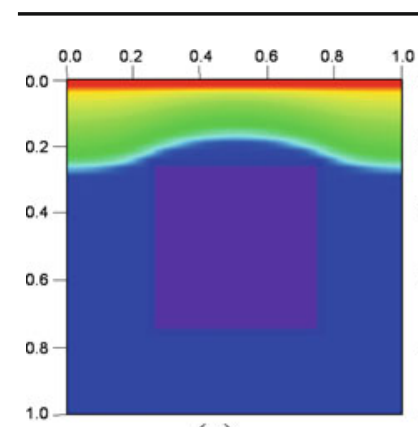

(a)

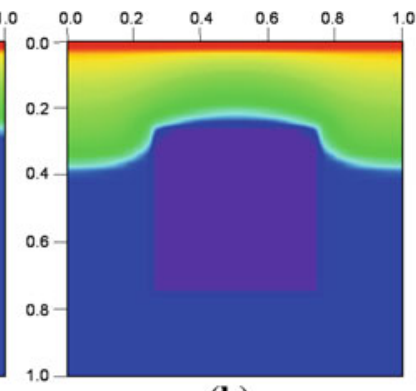

(b)

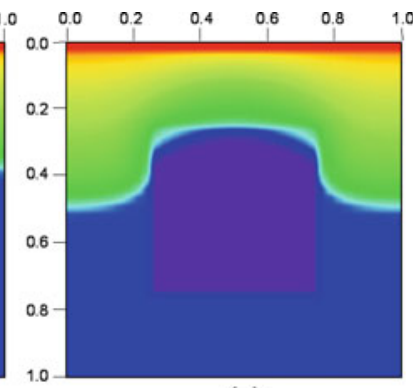

(c)

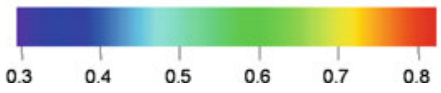

Fig. 14 Evolution of the saturation fields at three given times: a $t=0.22 \mathrm{~s}, \mathbf{b} t=0.33 \mathrm{~s}, \mathbf{c} t=0.44 \mathrm{~s}$ for two-dimensional flow in a nodular medium with $R e=3.1$. The Darcy-Forchheimer model is used with imposed pressure at the inlet

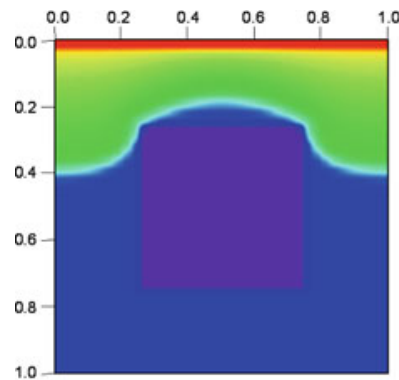

(a)

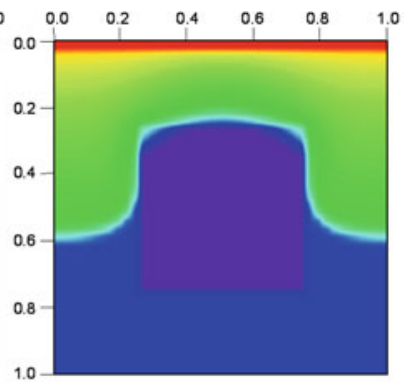

(b)

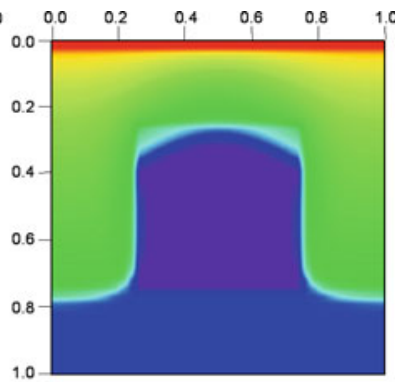

(c)

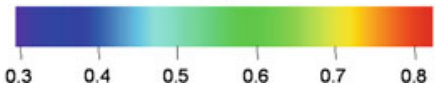

Fig. 15 Evolution of the saturation fields at three given times: $\mathbf{a} t=0.22 \mathrm{~s}, \mathbf{b} t=0.33 \mathrm{~s}$, c $t=0.44 \mathrm{~s}$ for two-dimensional flow in a nodular medium. Same case as in Fig. 14 using the Darcy model

\section{Conclusion}

On the basis of the generalized Darcy-Forchheimer model, a numerical tool has been developed for simulating two-phase inertial immiscible and incompressible flow in three-dimensional heterogeneous porous media. For clarity, the analysis of numerical results has been restricted to one and two dimensions. A convenient definition of the Reynolds number $(R e)$ allowing the quantification of inertial effects by comparing $R e$ with unity has been proposed. For one-dimensional flow in homogeneous porous media, under negligible capillary pressure assumption, the numerical tool has been validated by comparing the results to those obtained using a semi-analytical solution inspired from the Buckley-Leverett model but extended to take into account inertial effects. The nonlinear velocity-dependent part of the mobilities can be safely estimated using a fixed point iteration method.

Comprehensive simulation tests have been performed on 1D homogeneous and heterogeneous models of porous media as well as on 2D structures including layered and nodular media. Without introducing any particularity in the numerical approach, the study has been focused on imbibition only. In all the cases, it has been clearly highlighted, as expected, that 
breakthrough times are underestimated when inertial effects are neglected. For heterogeneous media, our conclusions are as follows:

- saturation change at the interface between two regions is well restored, as a result of contrasted petrophysical properties;

- in comparison to the Darcy regime, this saturation change can be completely modified and even reversed when inertial effects are taken into account;

- for 2D configurations, the slowdown of the saturation front induced by extra friction when inertial effects are taken into account is particularly pronounced in the region having the larger permeability, the front kinetic being only slightly affected in the smaller permeability region in comparison to the Darcy regime; and

- under certain conditions of capillary pressure and gradients of capillary pressure contrasts between two different regions, the saturation front at the interface between these two regions can be strongly affected by capillary cross flow, eventually leading to local trapping of the displaced fluid when the Reynolds number is small compared to unity. This effect can be inhibited when the Reynolds number is large enough, leading to a smooth saturation change.

These conclusions are only quantitatively dependent upon the choice of the capillary pressure curves and the inertial coefficients that were taken here in agreement with experimental correlations provided in the literature. This study provides a useful tool for future analysis and comprehension of macroscopic inertial two-phase flow in heterogeneous porous media.

Acknowledgments We wish to thank our students, Y. Benarafa and S. Delau, who participated in this study at its early stage.

\section{References}

Abdobal, S.: Ecoulements diphasiques en milieux poreux: Etude expérimentale des écoulements liquide-gaz et liquide-liquide à forts débits. PhD thesis, Institut National Polytechnique de Lorraine (2002)

Ahmadi, A., Quintard, M.: Calculation of large-scale properties for multiphase flow in random porous media. Iran. J. Sci. Technol. 9(1 Transaction A), 11-37 (1995)

Aziz, K., Settari, A.: Petroleum Reservoir Simulation. Elsevier Applied Science, London (1979)

Bear, J., Braester, C., Menier, P.C.: Effective and relative permeabilities of anisotropic porous media. Transp. Porous Media 2(3), 301-316 (1987)

Brooks, R.H., Corey, A.T.: Properties of porous media affecting fluid flow. J. Irrig. Drain. Div. Proc. ASCE 92(IR 2), 61-88 (1966)

Buckley, S.E., Leverett, M.C.: Mechanism of fluid displacement in sands. Trans. Am. Inst. Min. Petrol. Eng. 146, 107-116 (1942)

Chang, J., Yortsos, Y.: Effect of heterogeneity on Buckley-Leverett displacement. SPE paper 18798 (1989)

Chavent, G.: A new formulation of diphasic incompressible flows in porous media. Lecture Note in Math 503, pp. 258-270. Springer-Verlag, Berlin (1976)

Chen, Z., Huan, G., Li, B.: An improved IMPES method for two-phase flow in porous media. Transp. Porous Media 54, 361-376 (2004)

Corey, A.T.: The interrelation between gas and oil relative permeabilities. Prod. Mon. 19(1), 38-41 (1954)

Cornell, D., Katz, D.L.: Flow of gases through consolidated porous media. Ind. Eng. Chem. 45, 21452152 (1953)

Dale, M., Ekrann, S., Mykkeltveit, J., Virnovsky, G.: Effective relative permeabilities and capillary pressure for one-dimensional heterogeneous media. Transp. Porous Media 26, 229-260 (1997)

Ergun, S.: Fluid flow through packed columns. Chem. Eng. Prog. 48, 89-94 (1952)

Evans, E.V., Evans, R.D.: Influence of an immobile or mobile saturation on non-Darcy compressible flow of real gases in propped fractures. J. Petrol. Technol. 40(10), 1343-1351 (1988)

Evans, R.D., Hudson, C.S., Greenlee, J.E.: The effect of an immobile liquid saturation on the non-Darcy flow coefficient in porous media. J. SPE Prod. Eng. Trans. AIME 283, 331-338 (1987) 
Forchheimer, P.: Wasserbewegung durch boden. Z. Ver. Deutsch. Ing. 45, 1781-1788 (1901)

Fourar, M., Lenormand, R., Karimi-Fard, M., Horne, R.: Inertia effects in high-rate flow through heterogeneous porous media. Transp. Porous Media 60, 353-370 (2005)

Geertsma, J.: Estimating the coefficient of inertial resistant in fluid flow through porous media. SPE J. 14, 445-450 (1974)

Honarpour, M., Koederitz, L., Harvey, A.H.: Relative Permeability of Petroleum Reservoirs. CRC Press, Boca Raton (1986)

Hubbert, M.K.: Darcy's law and the field equation of the flow of underground fluids. Trans. SPE AIME 207, 222-239 (1956) (JPT)

Jamiolahmady, M., Danesh, A., Sohrabi, M., Duncan, D.: Measurement and modelling of gas condensate around rock perforation. Transp. Porous Media 63, 323-347 (2006)

Kalaydjian, F., Bourbiaux, J.M., Lombard, J.M.: Predicting gas-condensate reservoir performance: how flow parameters are altered when approaching producing wells. SPE paper 36715 (1996)

Katz, D.L., Lee, R.L.: Natural Gas Engineering, Production and Storage, Chemical Engineering Series. McGraw-Hill, New York (1990)

Khashan, S., Al-Amiri, A., Pop, I.: Numerical simulation of natural convection heat transfer in a porous cavity heated from below using a non-darcian and thermal non-equilibrium model. Int. J. Heat Mass Transf. 49(5-6), 1039-1049 (2006)

Kim, M., Park, E.: Fully discrete mixed finite element approximations for non-Darcy flows in porous media. Comput. Math. Appl. 38, 113-129 (1999)

Koederitz, L.F., Harvey, A.H., Honarpour, M.: Introduction to Petroleum Reservoir Analysis. Gulf, Houston (1989)

Lasseux, D., Whitaker, S., Quintard, M.: Determination of permeability tensors for two phase flow in homogeneous porous media: theroy. Transp. Porous Media 24(1), 103-137 (1996)

Lasseux, D., Ahmadi, A., Abbasian Arani, A.: Two-phase inertial flow in homogeneous porous media: a theoretical derivation of a macroscopic model. Transp. Porous Media 75, 371-400 (2008)

Lee, H.S., Catton, I.: Two-phase flow in stratified porous media. In: 6th Information Exchange Meeting on Debris Coolability, Los Angeles (1984)

Li, D., Engler, T.W.: Literature review on correlations of the non-Darcy coefficient. SPE paper 70015 (2001)

Lipinski, R.J.: A model for boiling and dryout in particle beds. Report SAND 82-0756 (NUREG/CR-2646) Sandia Labs (1982)

Liu, X., Civan, F., Evans, R.D.: Correlations of the non-Darcy flow coefficient. J. Can. Petrol. Technol. 34(10), 50-54 (1995)

Marle, C.M.: Cours de Production, Tome IV, Les Écoulements Polyphasiques En Milieu Poreux. Editions Technip, Paris (1972)

Mazaheri, A., Zerai, B., Ahmadi, G., Kadambi, J., Saylor, B., Oliver, M., Bromhal, G., Smith, D.: Computer simulation of flow through a lattice flow-cell model. Adv. Water Res. 28(12), 1267-1279 (2005)

Mei, C.C., Auriault, J.L.: The effect of weak inertia on flow through a porous medium. J. Fluid Mech. 222, 647-663 (1991)

Muskat, M.: The Flow of Homogeneous Fluids Through Porous Media. McGraw-Hill, New York (1937)

Saez, A.E., Carbonnell, R.G.: Hydrodynamic parameters for gas-liquid co-current flow in packed beds. AIChE J. 31, 52-62 (1985)

Sanchez, M., Luna, E., Medina, A., Méndez, F.: Simultaneous imbibition-heat convection process in a nonDarcian porous medium. J. Colloid Interface Sci. 288, 562-569 (2005)

Scheidegger, A.E.: The Physics of Flow Through Porous Media. University of Toronto Press, Toronto (1972)

Schulenberg, T., Muller, V.: An improved model for two-phase flow through beds of coarse particles. Int. J. Multiph. Flow 13, 87-97 (1987)

Skjetne, E., Auriault, J.L.: High-velocity laminar and turbulent flow in porous media. Transp. Porous Media 36(2), 131-147 (1999)

Tek, M.R., Coats, K.H., Katz, D.L.: The effect of turbulence on flow of natural gas through porous reservoirs. J. Petrol. Technol. Trans. AIME 222, 799-806 (1962)

Virnovsky, G., Friis, H., Lohne, A.: A steady-state upscaling approach for immiscible two-phase flow. Transp. Porous Media 54, 167-192 (2004)

Wahyudi, I., Montillet, A., Khalifa, A.O.A.: Darcy and post-Darcy flows within different sands. J. Hydraul. Res. 40(4), 519-525 (2000)

Wang, X., Thauvin, F., Mohanty, K.K.: Non-Darcy flow through anisotropic porous media. Chem. Eng. Sci. 54, 1859-1869 (1999)

Welge, H.J.: A simplified method for computing oil recovery by gas or water drive. Trans. Am. Inst. Min. Metall. Petrol. Eng. 195, 91-98 (1952) 
Whitaker, S.: The Forchheimer equation: a theoretical development. Transp. Porous Media 25(1), $27-61$ (1996) Whitney, D.D.: Characterization of the non-Darcy flow coefficient in propped hydraulic fractures. Master's thesis, University of Oklahoma (1988)

Wu, Y.S.: Non-Darcy displacement of immiscible fluids in porous media. Water Resour. Res. 37(12), 2943$2950(2001)$ 